\title{
Exploring designers' cognitive load when viewing different digital representations of spaces: A pilot study
}

\author{
Rongrong $\mathrm{Yu}^{1}$ and John Gero ${ }^{2}$ \\ ${ }^{1}$ Griffith University, Gold Coast, Australia \\ rongrong.yu@uon.edu.au \\ ${ }^{2}$ Krasnow Institute for Advanced Study, George Mason University, Fairfax, USA and \\ University of North Carolina at Charlotte, USA \\ john@johngero.com
}

\begin{abstract}
Do different representations of space evoke the same response in viewers? This paper reports on a pilot study exploring designers' cognitive load as they view different digital representation of spaces to determine the effect of the representations. The results reported in this paper are from a group of year 3 and year 4 architecture students from Harbin Institute of Technology in China who participated in the experiment. The two representational modalities in this study that participants were asked to view were a computer-generated hiddenline perspective and a digital photograph of the same space. Their physiological data were recorded by eye-tracking equipment, including participants' eye gaze trace location and pupil size. The same seven Area of Interests (AOI) were defined in each of the two representations. Results show statistically significant cognitive load change when the AOI focused on changes. The cognitive loads for the two representations were found not to be significantly different.
\end{abstract}

Keywords: designers' cognitive load· eye tracking• computational representation

\section{Introduction}

During any design process, designers develop their concepts with the assistance of visual representations [1]. With the development of digital design techniques the visual representation of digital models and images are now used broadly. However, the effect of digital representations on designers during the design process is not well understood. Do different modalities of representation evoke the same response in the viewer or different responses? With this pilot study we aim to produce evidence-based results about designers' physiological responses and cognitive load as they view different representation modalities of an architectural space.

Previous research has focused on how designers look at scenes using eye-tracking technology, which has suggested some characteristic behaviors. Kaufman and Richard [2] measured the eye fixation time allocated in several pre-defined parts of figures, the results show that the center of gravity is attractive as well as the edges and corners. 
Weber, Choi, and Stark [3] suggest that, with no a priori model in a figure, the attention would fixate at the center and that while the foreground was common for initial fixations, the eye did not typically scan the edges of interior space or rectilinearly-oriented contours. However, such research focused on comparing different arrangements of objects within a space, rather than different methods of representing the same spatial configuration. There is a lack of empirical evidence regarding designers' cognitive load when viewing different digital spatial representations. This is of interest as architects use multiple kinds of digital representations at different stages while they are designing. Whilst they have life experience with analog sketching and physical model-making, they do not have the same experience to draw on for different digital representations. This research is motivated by this gap in experiential understanding.

This study focuses on exploring designers' cognitive load when viewing different digital representations of the same architectural spaces and is part of a larger study on the veridicality of spatial representations. The results reported in this paper are from a group of year 3 and year 4 architecture student participants from Harbin Institute of Technology in China. The two representational modalities in this study that participants were asked to view were a computationally produced hidden-line perspective and a digital photograph of the same space. During the experiment, architecture students were required to look at each of the images for 20 seconds. Their physiological data were recorded by eye-tracking equipment that measured eye gaze trace location and pupil size. The same seven Area of Interests (AOI) were defined in each of the two representations. The relationship between the pupil size changes and the gaze changes in AOIs was then analyzed and discussed.

\section{Background}

\section{Computational Representation}

Design representation is a core issue in most design domains including product design, architecture and engineering. With the development of computational modeling digital design representation became possible during the design process. It is claimed that externalizing representations assists designers in both off-loading cognition and providing the possibility to interact with their external representations [1]. For example, BIM technology enables 3D (model check, design view, enhanced reality) and 4D visualization (i.e., the same plus time) [4]. Virtual reality (VR) can produce realistic virtual environments which provide designers with navigation possibilities [5]; augmented reality (AR) can enhance the user's perception by complementing the real world with 3D virtual objects in the same space [6]. These computer representation tools allow designers to more readily explore design ideas and assist with the concept development of their designs. However, there is a lack of understanding of the physiological effect of digital representations on designers.

Oliva et al. [7] studied the representation of the shape of visual space. In their study they critique the isovist theory of spatial perception for leaving out other 
characteristics of space such as texture, material and color that contribute to the human perception of space. They also propose a model called the 'spatial-envelope representation', which describes qualitatively the character and mood of a physical or pictorial space, represented by its boundaries (e.g., walls, floor, ceiling, and lighting) stripped of movable elements (e.g., objects and furnishing). Then they proposed a formal, computational approach to the capture of the shape of space as it would be perceived from an observer's vantage point [7-9]. The collection of properties describing a space in view is referred to as the spatial-envelope representation. This distinction in the characteristics that contribute to the human perception of space pertains to our research into representations of space that include or exclude some of those characteristics.

\section{Designers’ Cognitive Load Increase Pupil Size}

The concept of cognitive load is based on empirical evidence from experiments in psychology and physiology associated with cognitive activities [10, 11]. Cognitive load is the total amount of mental effort involved in using working memory. It has been found that there is a direct correlation between cognitive load and pupillary response. For instances, Hess and Polt [12] suggest that during the solving of simple multiplication problems, change in pupil size is related to mental activity. Just and Carpenter [13] show that different complexities of sentences affect pupil size when reading. Kahneman and Beatty (add to References and insert number here) found pupil size increases when participants remember more digits. Granholm et al. [11] suggest a higher cognitive load is associated with an increase in pupil dilation. Therefore, pupil diameter can be a measurement of mental workload and cognitive process [14]. This allows for this measure to be used to test whether different representations of architectural space entail different cognition loads. Different cognitive loads imply different responses by the viewer.

\section{Eye Tracking}

Early research on eye movement can be traced back to Buswell [15] who focused on the aesthetic impact of photographs of artwork, patterns and sculpture, particularly the layout patterns of advertisements. Kaufman and Richard [2] measured eye fixation times in several pre-defined parts of figures, the results show that the center of gravity is an attractor as are the edges and corners. Gould and Peeples [16] suggest that a subject's interpretation of a figure does not affect eye movements, which means that only "physical attributes" have influence on the eye movements. Kuchinke et al [20] suggested that longer fixation durations of eye movements positively correlate with memory performance. Valtchanov and Ellard found that visual information affects the cognitive load of viewers in multiple ways [21]. Torralba et al. [17] proposed visual attentional guidance through an experimental search task. Results of their study suggest that the context information plays an important role in object detection and observation. They also suggest some parts of the scene attract more attention than others. 
While the relationship between eye movement and perception of artworks has been investigated, there has been very little study on the role of eye movement in the perception of three-dimensional architectural space. One of the few studies on this topic was conducted by Weber, Choi, and Stark [3] in which they collected eye tracking data as participants were asked to look at three-dimensional models, or photographs of models, of architectural space. These models were constructed to collect data on the perception of the following architectural issues: empty space; symmetry vs. asymmetry; left and right reversed; obliquely-oriented elements; vista; and foreground. The research focused on comparing different arrangements of objects within a space, rather than different methods of representing the same spatial configuration. Their results showed that, with no priori model in a figure, the attention would fixate at the center; while the foreground was common for initial fixations, the eye did not typically scan the edges of interior space or rectilinearly-oriented contours; the objects on the left attract more attentions than the ones on the right. This confirmed the results by Arnheim [18]. The study also concluded that fixations did not vary significantly when viewing the physical model compared with a photograph of the model, with the exception of the foreground, which attracted greater attention in the physical model. Additionally, their results also suggest that there were significant differences between the fixations and saccades of architects and non-architects.

\section{EXPERIMENT}

We designed a pilot experiment with the aim of measuring physiological differences due to different modalities of representation of architectural spaces. We measured physiological response to two different digital representations of architectural spaces. In this paper we report results from measuring pupil dilation related to eye movement and saccades. In this experiment, 10 third and fourth year architecture students with similar backgrounds at Harbin Institute of Technology (HIT) in China participated in this pilot study. Given the homogeneity of participant cohort 10 participants are sufficient to produce statistically reliable results. The selection of third year and fourth year architecture students was because design students at this stage already have basic aesthetic and design sense, which is appropriate for the current study. During this experiment participants' eye movements constantly changed, which produced large amount of data - up to 6,000 eye movement data points were captured for each participant. This extensive data enabled detailed analysis of designers' eye movement and pupillary behavior.

During the experiment participants were initially asked to complete demographic questions regarding their gender, age and first language. Then they were required to look at two images shown on a screen, Figure 1. Figure 2 shows the two images used: the first one (R1) is a computer generated perspective drawing of a set of architectural spaces, and the second (R0) is a digital photograph of the same spaces when built. The reason for comparing these two representations is that - firstly, the modality of representation may affect the cognitive load of the viewer, secondly, the color in the 
digital photograph may affect designers' cognitive attention; and thirdly, the complexity level of the areas in the image may be affected by the color.

The participants' eye saccades and fixations data were recorded by an eye-tracking system (Tobii studio) as they looked at the images. Each of the images was shown for 20 seconds with a few seconds for recalibration in between them. Half of participants were first shown R1, then R0. The other half were first shown R0, then R1. After each image session there was a questionnaire session. Data collected included eye fixations, saccades and pupil dilation.

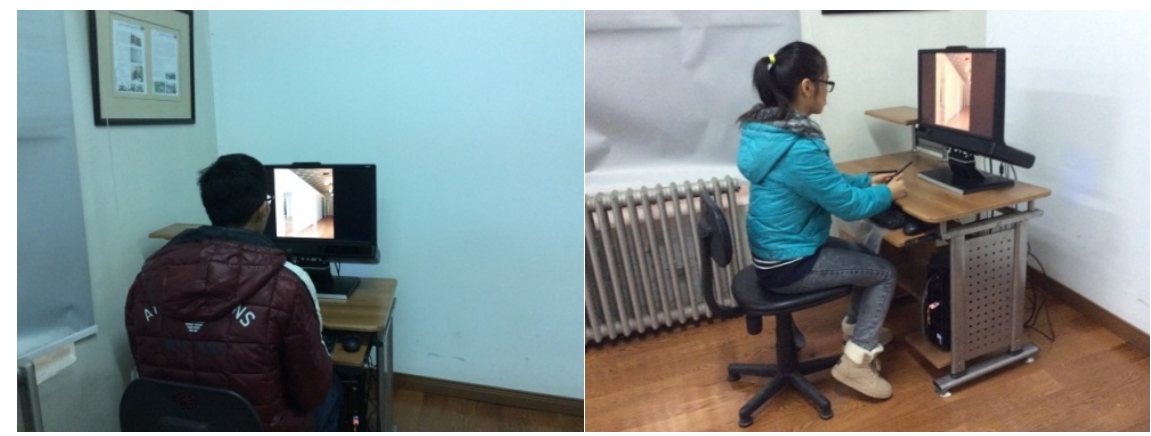

Fig. 1. Two views of the experiment setup
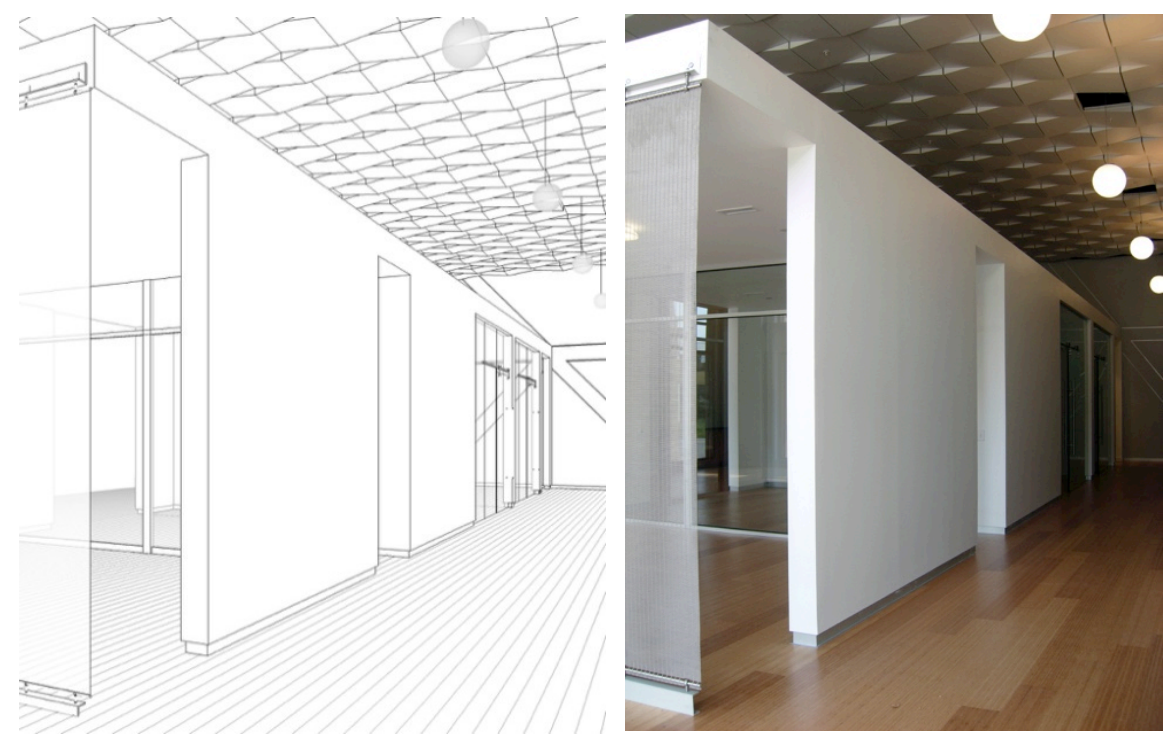

Fig. 2. Left: Computer generated hidden-line perspective of a set of architectural spaces (labeled R1 in the experiment). Right: Digitized photograph of the same spaces (labeled R0 in the experiment) 
To identify the characteristics of participants' response to the spatial representation, we identified 7 Areas of Interest (AOIs) in the visual scene, Figure. 3. Each AOI defines an area that we wanted to gather data about. AOIs defined the three doorways, the two wall surfaces between them, the terminus of the corridor and the ceiling.

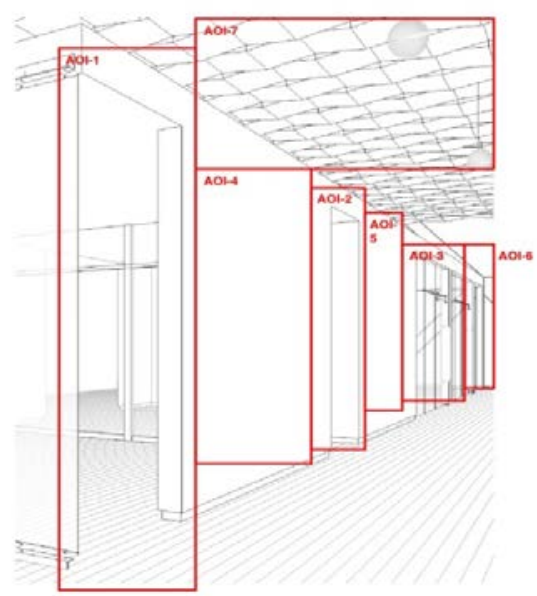

Fig. 3. The seven Areas of Interest labeled AOI 1 through AOI 7 in R1, they occupy the same positions in R0.

\section{RESULTS}

During the experiment, we captured eye movement data for each AOI for each gaze, and pupil size of both eyes. Table 1 shows the pupil size changes with AOI of each participant when they looked at the two images (R1 and R0). The red line represents the AOI focused on, while the blue line represents the pupil size changes. When a participant looked at any location outside the 7 AOIs that was coded as 0. Next, we analyzed the correlation between AOI changes and pupil size changes. The method of analysis is that each time a participant's AOI changes, we measured the mean pupil size of the two eyes for the 30 segments before and after the changes. This gives us four metrics, which are compared using an unpaired t-test, with a threshold of $\mathrm{p}<0.05$ considered as statistically significance.

Table 2 shows the averages of the four metrics for the data of the 7 AOIs in Image R1 and R0. From this analysis, we found on average $84.0 \%$ of the pupil size change are significantly related to AOI changes when designers look the line drawing image (R1). While they look at the digital color photo, this value is $83.1 \%$ (R0). We can infer that pupil size change when the AOI focused on changes is statistically significant in both the line drawing images and the digital photo. The pupil size changes are related to their cognitive load, therefore, results confirm that designers' cognitive load are associated with the AOIs they looked at. 
Table. 1. Plots of AOI focused on and pupil diameter for three of the participants. The red line is the AOI focused on at that time (the vertical axis is a nominal number whose position has no meaning), the blue line is the average pupil diameter.

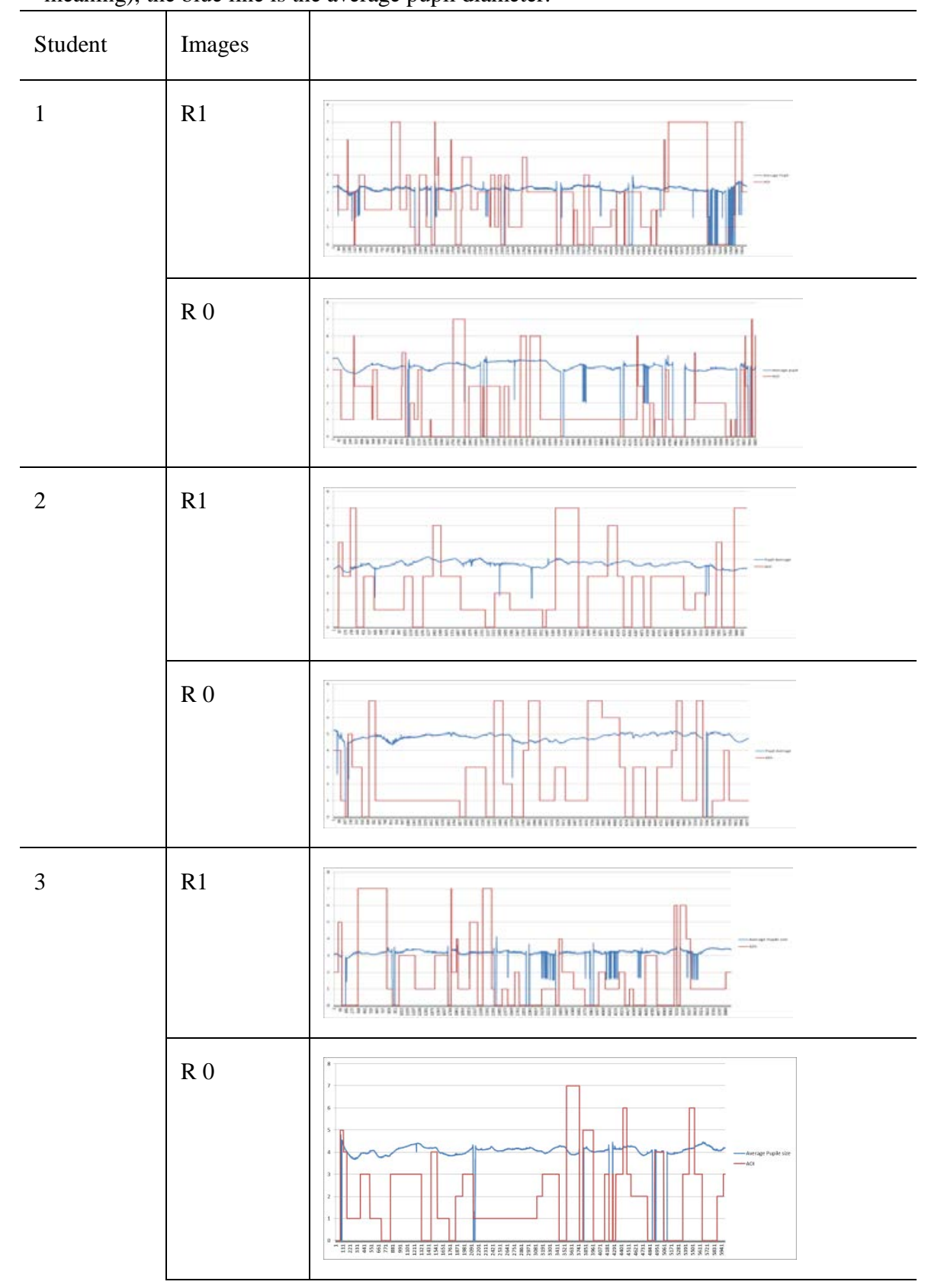


Table. 2. Percentage of significant pupil size change when moving from AOI to AOI $(\mathrm{p}<0.05)$

\begin{tabular}{c|c|c}
\hline & $\begin{array}{c}\text { Percentage of significant } \\
\text { pupil size changes } \\
\text { Image R1 }\end{array}$ & $\begin{array}{c}\text { Percentage of significant } \\
\text { pupil size changes } \\
\text { Image R0 }\end{array}$ \\
\hline Student 1 & 70.2 & 82.1 \\
\hline Student 2 & 84.9 & 75.0 \\
\hline Student 3 & 82.9 & 92.1 \\
\hline Student 4 & 66.7 & 59.6 \\
\hline Student 5 & 95.4 & 92.9 \\
\hline Student 6 & 76.6 & 76.3 \\
\hline Student 7 & 94.4 & 95.5 \\
\hline Student 8 & 82.8 & 85.4 \\
\hline Student 9 & 95.2 & 84.3 \\
\hline Student 10 & 91.3 & 87.5 \\
\hline Average & 84.0 & 10.1 \\
\hline SD & 9.8 &
\end{tabular}

From an unpaired t-test of pupil size change of Image R1 and Image R0, there is no significant difference in cognitive load due to representational modality.

From the same pilot study, Table 3 shows the eye movements results for the same 30 HIT students. In comparing Image R1 and Image R0 there were differences in three of the four eye movement measurements: time to view, time viewed, fixations and revisits. Time to first view, time viewed and fixations were all statistically significantly different when viewing the computer-generated line drawing perspective compared to viewing the digital color photograph for Area of Interest 1, which is the first AOI to be viewed in the digital photograph but is only the third AOI to be viewed in the line drawing perspective. Interestingly, AOI-1 had the highest percentage of time viewed for both modalities, whilst there was a lack of congruence for the other three measures.

Table. 3. T-tests of Image R1 compared with Image R0 for students from HIT [19]

\begin{tabular}{c|c|c|c|c|c|c|c}
\hline & \multicolumn{7}{c}{ p values } \\
\hline AOI & 1 & 2 & 3 & 4 & 5 & 6 & 7 \\
\hline $\begin{array}{c}\text { Time to First } \\
\text { View (secs) }\end{array}$ & $0.001^{*}$ & 0.204 & $0.030^{*}$ & 0.633 & 0.400 & 0.353 & $0.029 *$ \\
\hline $\begin{array}{c}\text { Time Viewed } \\
(\%)\end{array}$ & $0.028^{*}$ & 0.267 & $0.009 *$ & 0.633 & 0.154 & 0.067 & $0.026^{*}$ \\
\hline Fixations & $0.003^{*}$ & 0.960 & 0.873 & 0.633 & $0.015^{*}$ & 0.359 & 0.631 \\
\hline Revisits & $0.002^{*}$ & 0.751 & 0.667 & 0.633 & $0.007^{*}$ & 0.820 & 0.591 \\
\hline
\end{tabular}

$* \mathrm{p}<0.05$ 
Even though the cognitive load of both digital representations is the same there are significant other physiological differences between the digital photo image and line drawing image when being viewed.

\section{CONCLUSION}

This paper presents the results of eye-tracking measurements of pupil diameter of architecture students looking at two different digital representations of the same spaces. As expected the results of this study suggest that architectural students' pupil size change is significantly related to changes of their focus area for both the computer generated perspective drawing and digital photograph images. Pupil diameter directly correlates with the viewers' cognitive load. Since the pupil size change is related to human cognitive working load, we can infer that students' cognitive load is related to changes as they focused on different AOIs. However, there was no statistical difference in the changes in cognitive load between the two different representation modalities. Since the earlier result in Gero et al. [19] showed significant differences in where they looked and for how long they looked, the results in this paper imply that cognitive load is not directly related to representational modality. This is a potentially important result, if confirmed by future experiments to be generally applicable, as it is unexpected that at this level of cognition the two representational modalities are similar. Since the earlier results have shown that there are significant differences in eye gaze fixations, this result implies that not all cognitive activities are affected by representational modality. Results of this study contribute to the current understanding of the relationship between designers' cognitive load with their physical reactions, which provides new perspectives for bridging design studies and cognitive science. Future work of this study is to expand the sample size to achieve more statistically robust results, to explore the relationship between students' eye movement and their cognitive design thinking, and to increase the number of representational modalities with the goal of producing an understanding

for designers of the implications of the use of representations both for themselves and for their clients.

\section{Acknowledgement}

This research was carried out under UNCC’s IRB Approval for Protocol 14-03-17.

\section{References}

1. Schön, D. and G. Wiggins: Kinds of seeing and their functions in designing. Design Studies. 13(2), 135-156, (1992)

2. Kaufman, L. and W. Richard: Spontaneous fixation tendencies for visual forms. Perception and Psychophysics. 585-88, (1969) 
3. Weber, R., Y. Choi, and L. Stark: The Impact of Formal Properties on Eye Movement During the Perception of Architecture. Journal of Architectural Planning and Research. 19(1), 57-68, (2002)

4. Eastman, C.M.: BIM Handbook: A Guide to Building Information Modeling for Owners, Managers, Designers, Engineers and Contractors. Wiley (2008)

5. Wang, W., et al.: Creation dependencies of evolutionary artefact and design process knowledge. Journal of Engineering Design. 24(9), 681-710, (2013)

6. Morrison, A., et al.: Collaborative use of mobile augmented reality with paper maps. Computers \& Graphics. 35(4), 789-799, (2011)

7. Oliva, A., S. Park, and T. Konkle: Representing, perceiving and remembering the shape of visual space. . In: L.R. Harris and M. Jenkin, Editor^Editors (eds.) Vision in 3D Environments. pp. 308-339.Cambridge University Press, Cambridge. (2011)

8. Oliva, A. and A. Torralba: Modeling the Shape of the Scene: A Holistic Representation of the Spatial Envelope. International Journal of Computer Vision. 42(3), 145-175, (2001)

9. Oliva, A. and A. Torralba: Building the gist of a scene: the role of global image features in recognition. Progress in Brain Research. 15523-36, (2006)

10. Buettner, R.: Cognitive workload of humans using artificial intelligence systems: Towards objective measurement applying eye-tracking technology. In: I.J. Timm, and Thimm, Matthias, (eds.) KI 2013: Advances in Artificial Intelligence. KI 2013: 36th German Conference on Artificial Intelligence. pp. 37-48.Springer, Koblenz, Germany. (2013)

11. Granholm, E., et al.: Pupillary responses index cognitive resource limitations. Psychophysiology 33(4), 457-461, (1996)

12. Hess, E.H. and J.M. Polt: Pupil size in relation to mental activity during simple problemsolving. Science. 143(3611), 1190-1192, (1964)

13. Just, M.A. and P.A. Carpenter: The intensity dimension of thought: Pupillometric indices of sentence processing. Canadian Journal of Experimental Psychology. 47(2), 310-339, (1993)

14. Holmqvist, K., et al.: Eye Tracking: A Comprehensive Guide to Methods and Measures. Oxford, Oxford University Press ( 2011)

15. Buswell: How people look at pictures: A study of the psychology of perception in art. Chicago, University of Chicago Press (1935)

16. Gould, D. and R. Peeples: Eye movements during visual search and discrimination of meaningless symbol and object patterns. Journal of Experimental Psychology. 8551-55, (1970) 17. Torralba, A., et al.: Contextual Guidance of Eye Movements and Attention in Real-World Scenes: The Role of Global Features in Object Search. Psychological Review. 113(4), 766 786, (2006)

18. Arnheim, R.: The power of the center. Berkeley, University of California Press (1985)

19. Gero, J., J. Shields, and R. Yu: How veridical are computer generated representations? -The Effect of Presentation Modality on Physiological Response, in The 21st International Conference on Computer-Aided Architectural Design Research in Asia (CAADRIA 2016). 2016. p. 861-870.

20. Kuchinke, L., et al.: The processing and integration of map elements during a recognition memory task is mirrored in eye-movement patterns. Journal of Environmental Psychology. 47213-222, (2016)

21. Valtchanov, D. and C.G. Ellard: Cognitive and affective responses to natural scenes: Effects of low level visual properties on preference, cognitive load and eye-movements. Journal of Environmental Psychology. 43184-195, (2015) 